\title{
What clocks tell us about the neural correlates of spatial imagery
}

Citation for published version (APA):

Trojano, L., Linden, D., Formisano, E., Goebel, R., Sack, A., \& Di Salle, F. (2004). What clocks tell us about the neural correlates of spatial imagery. European Journal of Cognitive Psychology, 16(5), 653-672. https://doi.org/10.1080/09541440340000510

Document status and date:

Published: 01/01/2004

DOI:

10.1080/09541440340000510

Document Version:

Publisher's PDF, also known as Version of record

Document license:

Taverne

Please check the document version of this publication:

- A submitted manuscript is the version of the article upon submission and before peer-review. There can be important differences between the submitted version and the official published version of record.

People interested in the research are advised to contact the author for the final version of the publication, or visit the DOI to the publisher's website.

- The final author version and the galley proof are versions of the publication after peer review.

- The final published version features the final layout of the paper including the volume, issue and page numbers.

Link to publication

\footnotetext{
General rights rights.

- You may freely distribute the URL identifying the publication in the public portal. please follow below link for the End User Agreement:

www.umlib.nl/taverne-license

Take down policy

If you believe that this document breaches copyright please contact us at:

repository@maastrichtuniversity.nl

providing details and we will investigate your claim.
}

Copyright and moral rights for the publications made accessible in the public portal are retained by the authors and/or other copyright owners and it is a condition of accessing publications that users recognise and abide by the legal requirements associated with these

- Users may download and print one copy of any publication from the public portal for the purpose of private study or research.

- You may not further distribute the material or use it for any profit-making activity or commercial gain

If the publication is distributed under the terms of Article $25 \mathrm{fa}$ of the Dutch Copyright Act, indicated by the "Taverne" license above, 


\section{European Journal of Cognitive Psychology}

\section{What clocks tell us about the neural correlates of spatial imagery}

\section{Luigi Trojano , David Linden, Elia Formisano , Rainer Goebel , Alexander Sack \& Francesco Di Salle}

To cite this article: Luigi Trojano, David Linden , Elia Formisano, Rainer Goebel , Alexander Sack \& Francesco Di Salle (2004) What clocks tell us about the neural correlates of spatial imagery, European Journal of Cognitive Psychology, 16:5, 653-672, DOI: 10.1080/09541440340000510

To link to this article: https://doi.org/10.1080/09541440340000510

册Published online: 03 Jun 2010.

Submit your article to this journal ๘

山 Article views: 59

Q View related articles ¿

4 Citing articles: 1 View citing articles 준 


\title{
What clocks tell us about the neural correlates of spatial imagery
}

\author{
Luigi Trojano \\ Department of Psychology, Second University of Naples, Caserta, \\ and Salvatore Maugeri Foundation, IRCCS, Institute of Telese, Italy \\ David E. J. Linden \\ Max-Planck-Institut für Hirnforschung, and Department of Psychiatry, \\ Laboratory for Neurophysiology and Neuroimaging, \\ Johann Wolfgang Goethe-Universität, Frankfurt, Germany, \\ and School of Psychology, University of Wales, Bangor, UK

\section{Elia Formisano and Rainer Goebel} \\ Department of Cognitive Neuroscience, Faculty of Psychology, \\ Maastricht University, The Netherlands
}

\author{
Alexander T. Sack \\ Department of Psychiatry, Laboratory for Neurophysiology and Neuroimaging, \\ Johann Wolfgang Goethe-Universität, Frankfurt, Germany \\ Francesco Di Salle \\ Department of Neurological Sciences, Division of Neuroradiology, \\ Federico II University, Naples, Italy
}

\begin{abstract}
We review a series of experimental studies aimed at answering some critical questions about the neural basis of spatial imagery. Our group used functional magnetic resonance imaging (fMRI) to explore the neural correlates of an online behaviourally controlled spatial imagery task without need for visual presentation - the mental clock task. Subjects are asked to imagine pairs of times that are presented acoustically and to judge at which of the two times the clock hands form the greater angle. The cortical activation elicited by this task was contrasted with that obtained during other visual, perceptual, verbal, and spatial imagery tasks in several block design studies. Moreover, our group performed an event-related fMRI study on the clock task to investigate the representation of component cognitive processes in spatial imagery. The bulk of our findings demonstrates that
\end{abstract}

Correspondence should be addressed to Luigi Trojano, Department of Psychology, Second University of Naples, Via Vivaldi 43, 81100 Caserta, Italy. Email: luigi.trojano@unina2.it

(C) 2004 Psychology Press Ltd 
cortical areas in the posterior parietal cortex (PPC), along the intraparietal sulcus, are robustly involved in spatial mental imagery and in other tasks requiring spatial transformations. PPC is bilaterally involved in different kinds of spatial judgement. Yet the degree to which right and left PPC are activated in different tasks is a function of task requirements. From event-related fMRI data we obtained evidence that left and right PPC are activated asynchronously during the clock task and this could reflect their different functional role in subserving cognitive components of visuospatial imagery.

\section{A BRIEF OVERVIEW OF THE NEUROPSYCHOLOGY OF SPATIAL MENTAL IMAGERY}

Many tasks have been used to study the ability to visualise objects in the mind. Some of them require subjects to represent mentally visual features of objects, while others explicitly require the processing of spatially coded information. In the neuropsychological literature, several brain-lesioned patients have been reported with selective deficits in the performance of visual or spatial imagery tasks. Levine, Warach, and Farah (1985) and Farah, Hammond, Levine, and Calvanio (1988) described a patient who had a specific impairment in performing visual imagery tasks concerning the colour, size, and shape of objects. This patient was, for example, unable to tell whether certain animals have long or short tails or to compare the outlines of the borders of the American states. Yet he could perform spatial imagery tasks like mental rotation of letters or 3-D abstract figures and knew perfectly the location of the states. Levine et al. (1985) had already reported a patient with the complementary imagery deficit, but an extensive description of a patient affected by a selective impairment of spatial imagery was offered by Luzzatti, Vecchi, Agazzi, Cesa-Bianchi, and Vergani (1998). This patient could draw from memory or describe objects and animals correctly, and answer questions about visual features of objects, but was unable to describe spatial relationships among elements of complex objects or among streets and squares of her hometown. Similarly, she failed in spatial imagery tasks that required the construction of imagined matrices or spatial configurations.

This neuropsychological evidence for a double dissociation between visual and spatial imagery tasks suggests that distinct cognitive processes are involved in the two kinds of imagery. This distinction parallels that established in the visual perception domain into an occipitotemporal (ventral) pathway responsible for object identification and an occipital-parietal (dorsal) pathway responsible for spatial processing (Ungerleider \& Mishkin, 1982). Nonetheless, neuroanatomical findings in these patients did not provide stringent clues for the neural basis of spatial mental imagery. In fact, the patient with a selective impairment in visual imagery tasks described by Farah et al. (1988) had widespread bilateral temporo-occipital and right inferofrontal lesions, whereas the patients with selective impairment in spatial imagery tasks had bilateral parieto- 
occipital lesions (Levine et al., 1985) or a cortical atrophy that was more prominent in the right temporal lobe (Luzzatti et al., 1998).

\section{FUNCTIONAL NEUROIMAGING STUDIES OF SPATIAL MENTAL IMAGERY}

The issue of the neural basis of spatial imagery has been tackled by means of modern neuroimaging techniques in different experimental paradigms. In their recent empirical review of neuroimaging studies, Cabeza and Nyberg (2000) list several papers on spatial imagery, most of which employed visual presentations of stimuli that had to be mentally rotated to comply with task requirements (Alivisatos \& Petrides, 1997; Cohen et al., 1996; Kosslyn, DiGirolamo, Thompson, \& Alpert, 1998). Only a few studies investigated the entire process of spatial image generation and processing in the absence of visual stimulation. In the first (Mellet et al., 1995), subjects mentally explored a map memorised in previous learning sessions. In a second study (Mellet, Tzourio, Denis, \& Mazoyer, 1996), subjects mentally assembled objects from spatial instructions they had heard. A third study required subjects to perform a mental navigation along routes previously memorised through a walk in the real environment (Ghaem et al., 1997).

In their recent critical meta-analysis of functional studies on imagery, Thompson and Kosslyn (2000) concluded that the activation patterns during imagery tasks depend strongly on the kind of task used, the type of the experimental paradigm, and the neuroimaging method. When the task requires spatial operations or when mental images have to be transformed, posterior parietal regions are likely to be activated, while primary visual cortex activation would be expected when tasks require detailed, high-resolution mental images. For instance, activation of posterior parietal cortex (PPC) has been observed in conjunction with the spatial transformation of visually presented stimuli (Alivisatos \& Petrides, 1997; Cohen et al., 1996). In a recent PET study of mental rotation (Alivisatos \& Petrides, 1997), right PPC was seen to participate in the processing of mirror images of letters or digits. Activation of the superior parietal lobule in both hemispheres has been described in an fMRI study of the analysis of spatially transformed words and phrases, which also distinguished this spatial transformation-related activation from general attentional effects (Goebel, Linden, Lanfermann, Zanella, \& Singer, 1998b). The construction of three-dimensional mental images from auditory instructions, as studied by PET (Mellet, Tzourio, Crivello, Joliot, Denis, \& Mazoyer, 1996), involved a distributed system of frontal, occipital, and parietal areas. The parietal activation, however, was most prominent in the right precuneus and supramarginal gyrus and did not involve the PPC.

The majority of earlier studies could not monitor subjects' performances online during the execution of the task, and some involved visual presentation of 
stimuli, generating the possible confound of saccade-related activation in the parietal lobe (Milner \& Goodale, 1995). Our group used fMRI to explore the neural correlates of an online behaviourally controlled spatial imagery task without need for visual presentation. In some subjects we also controlled eye movements during the scanning session. The experimental task was derived from the mental clock task (Grossi, Angelini, Pecchinenda, \& Pizzamiglio, 1993; Grossi, Modafferi, Pelosi, \& Trojano, 1989; Paivio, 1978). Subjects are asked to imagine pairs of times that are presented acoustically and to judge at which of the two times the clock hands form the greater angle. Paivio (1978) demonstrated that when subjects have to judge the difference between the angles formed by the hour and the minute hand on an imagined clock face, they report using imagery and show a symbolic distance effect: reaction time increases as angular size difference decreases. The clock task is particularly suitable for the functional magnetic resonance imaging (fMRI) investigation of mental imagery because it involves a behavioural control that can be performed online during scanning and has the specific advantage that pairs of digital clock times can be presented visually to compare performance and cerebral activation between an imagery and a closely matched visual perceptual condition. Moreover, the task permits the assessment of imagery abilities separately for each visual hemifield. The clock task has already been used in neuropsychological studies on brainlesioned patients with selective imagery defects or with neglect-related spatial deficits (Grossi et al., 1989, 1993).

We planned a series of experimental studies aimed to clarify the pattern of cortical activation during the execution of the mental clock task. In particular, we aimed at answering a series of critical questions about the neural basis of spatial imagery.

\section{WHICH CORTICAL NETWORK IS INVOLVED IN SPATIAL IMAGERY AND IS IT SHARED WITH VISUAL PERCEPTION?}

In the first study (Trojano et al., 2000) the mental clock task was used in two experiments that addressed the cortical network involved in spatial imagery and its relationship to perceptual visuospatial processing. The two experiments provided converging evidence for the involvement of the PPC of both cerebral hemispheres in spatial imagery. Four healthy subjects participated in the second experiment, where we compared the activity during the mental clock task (imagery) to the same operation performed on visually presented clocks (perception) and to a nonspatial control task (syllable counting), whose attentional load proved comparable to that of the imagery task. The MR scanner used for imaging was a $1.5 \mathrm{~T}$ whole body superconducting system (MAGNETOM Vision, Siemens Medical Systems, Erlangen, Germany) equipped with a standard head coil, an active shielded gradient coil $(25 \mathrm{mT} / \mathrm{m})$ and Echo Planar (EPI) 
sequences for ultrafast MR imaging. For functional imaging, we used a BOLD (blood oxygenation level-dependent) sensitive single shot EPI sequence (echo time, $\mathrm{TE}=66 \mathrm{~ms}$; flip angle, $\mathrm{FA}=90^{\circ}$; matrix size $=128 \times 128$, voxel dimensions $=1.4 \mathrm{~mm} \times 1.4 \mathrm{~mm} \times 4 \mathrm{~mm}$ ) with an interscan temporal spacing of $5 \mathrm{~s}$. Stimuli were presented in a block design.

In the mental clock task, subjects had to imagine pairs of analogue clock faces based on the times that were presented verbally by the examiner (e.g., 9.30 and 10.00; ISI $=1 \mathrm{~s}$ ), and to judge at which of the two times the clock hands form the greater angle (imagery condition). In half stimuli, hours had to be imagined on the right half of the clock face (e.g., 3.00), and in the remaining half hours occupied the left half of the dial (e.g., 9.00); presentation order was randomised. During the syllable counting condition, we asked subjects to count the syllables of each pair of times and to report whether the total syllable number was odd or even. In the perception condition we used pairs of analogue clock faces; the clock faces of each pair were generated on a computer screen and projected one at a time (ISI $=1 \mathrm{~s}$ ) in the central visual field.

In all the experimental conditions, half of the subjects had to push a button with their right index finger to choose the first stimulus of each pair as the correct response, or their left index finger to choose the second stimulus; in the remaining subjects, response modality was reversed. Subjects' responses were registered by an optic fibre answer box and analysed for speed and accuracy. During the imagery and perception condition, material and response modality were the same. In the latter case, however, the pairs of times were presented visually to the subject while in the imagery condition they had to be visualised mentally. In the syllable counting condition, material, presentation and response modality were the same as in the imagery task, but here a verbal-phonological judgement was required. Subjects were asked to keep their eyes open during the scanning session and foveate a fixation cross in order to avoid eye movements.

Data analysis, registration, and surface-based visualisation were performed with the fMRI software package BrainVoyager (Goebel \& Singer, 1999). The statistical analysis of the BOLD signal (for review see Di Salle et al., 1999) time courses was based on the general linear model of the experiment. In this approach, each experimental condition (i.e., imagery, perception, syllable counting) is considered an effect of interest. The corresponding time points, convolved with a haemodynamic response function modelling the characteristics of the BOLD response, constitute the predictors of the model. The visualisation tools of the BrainVoyager software permit the reconstruction of the cortical surface of the subject's brain on the basis of a high-resolution 3-D anatomical MR data set. Colour-coded statistical maps $\left(p^{\prime}<10-3\right.$ corrected $)$ can then be visualised conventionally on individual slices through the brain or on surface representations. The display of functional maps on inflated or flattened hemispheres allows the topographic representation of the three-dimensional pattern of cortical activation without loss of the lobular structure of the telencephalon 
(Goebel, Khorram-Sefat, Muckli, Hacker, \& Singer, 1998a; Linden, 2002; Linden et al., 1999).

Behavioural results showed that the imagery (mean correct response time: $2517 \pm 860 \mathrm{~ms} ; 45.5 \pm 6.4$ correct responses out of 48 trials) and syllable counting conditions (mean correct response time: $2940 \pm 1039 \mathrm{~ms} ; 39.5 \pm 4.9$ correct responses) had equivalent processing load (no significant differences on MannWhitney test), while the perception condition proved to be significantly simpler than the others, both for response accuracy (47.1 \pm 0.8 correct responses) and reaction times (mean correct response time: $1433 \pm 618 \mathrm{~ms}$ ). Multiple regression analysis of the BOLD time course revealed a significantly higher activation of the intraparietal sulcus (IPS) region of the posterior parietal cortex (PPC) for the execution of the mental clock task compared to the syllable counting condition (Figure 1A). The comparison between the perception and baseline conditions showed an activation of posterior parietal cortex very similar to that observed during the imagery condition. However, in the contrast between the imagery and perception conditions (Figure 1B) no activation was evident in PPC. In the imagery condition increased activity was present in right prefrontal cortex and in mesial frontal areas bilaterally, while occipital and inferior temporal areas were activated bilaterally in the perception condition (see also Table 1).

The most striking result of this study is that cortical activation (as measured by an increase of the fMRI BOLD signal) during the mental clock task was most prominent in the posterior parietal lobes of both hemispheres. This activation can be regarded as specific for the visuospatial operations because it was observed in both visuospatial tasks (in the imagery and perceptual domain), but not in a nonvisuospatial control task that involved the same acoustically presented material and was of equivalent difficulty as the imagery task. The direct contrast between the perception and imagery conditions (i.e., the clock task performed on visually presented and mentally imagined material, respectively) yielded no significant activation differences in the PPC. This indicates that this area is recruited to a comparable extent for visuospatial imagery and perceptual processing.

Interestingly, no similar overlap of activation was observed in occipitotemporal cortex. The perception condition (spatial matching of visually presented clocks), compared to the fixation baseline, yielded very prominent bilateral activation of the inferior temporal and the inferior and lateral occipital lobes. This occipitotemporal activation was still observed when the perception condition was contrasted with the imagery condition. These areas, which include the area LO and the fusiform gyri, have been shown to be involved in the processing of visual objects (Malach et al., 1995). They are assumed to form part of the ventral pathway of visual processing (Ungerleider \& Mishkin, 1982), while the posterior parietal cortex has been assigned a role in the dorsal pathway responsible for the processing of visual motion and space (Goebel et al., 1998b; Ungerleider \& Haxby, 1994). 


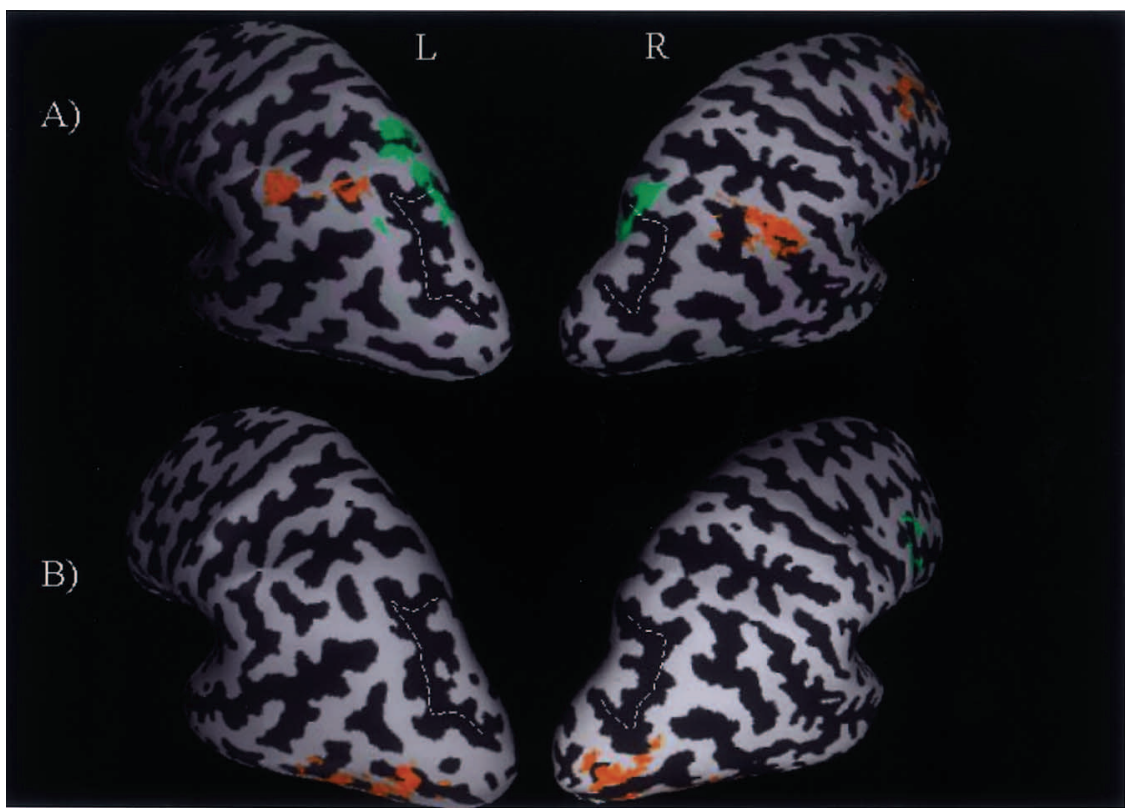

Figure 1. Statistical results of the first block design study of the mental clock task (Trojano et al., 2000) were visualised through projecting 3-D statistical maps on inflated surface reconstructions of the cortical sheet (posterior view, the dotted lines indicate the IPS). For significantly activated voxels, the relative contribution $\mathrm{RC}$ between two selected sets of conditions in explaining the variance of a voxel time course were computed as $R C=(b 1-b 2) /(b 1+b 2)$ where bi is the sum of the estimates of the standardised regression coefficients of all conditions included in set 1 . The RC index was visualised with a red-green pseudocolour scale. An RC value of 1 (red) indicates that a voxel time course is solely explained with predictor set 1 , whereas an $\mathrm{RC}$ value of -1 (green) indicates that a voxel time course is explained solely with predictor set 2 . A RC value of 0 indicates that a voxel time course is explained with an equal contribution of both predictor sets. In the relative contribution maps, only RC values greater than 0.7 were visualised. (A) The relative contribution of the imagery (green) and syllable counting (red) predictors to the explanation of the variance of the cortical BOLD signal change. Note that imagery-related activation is apparent along the IPS bilaterally while syllable counting involved the inferior parietal cortex (IPL) bilaterally and the right DLPFC. (B) An analogous relative contribution map for the imagery (green) and perception (red) predictors. Note that no imagery-related activation was observed in occipitotemporal areas.

The white dotted line corresponds to the intraparietal sulcus and its main branches. Reprinted with permission of Oxford University Press (Trojano et al., 2000).

Inferior temporal areas were also found to be involved in visual mental imagery, particularly when object properties had to be encoded as in the case of imagery of faces and places (O'Craven \& Kanwisher, 2000). In our study of spatial imagery, however, the ventral pathway was recruited exclusively during the perceptual condition and convergence of the cortical processing streams for visuospatial processing in perception and imagery was only observed at the level 


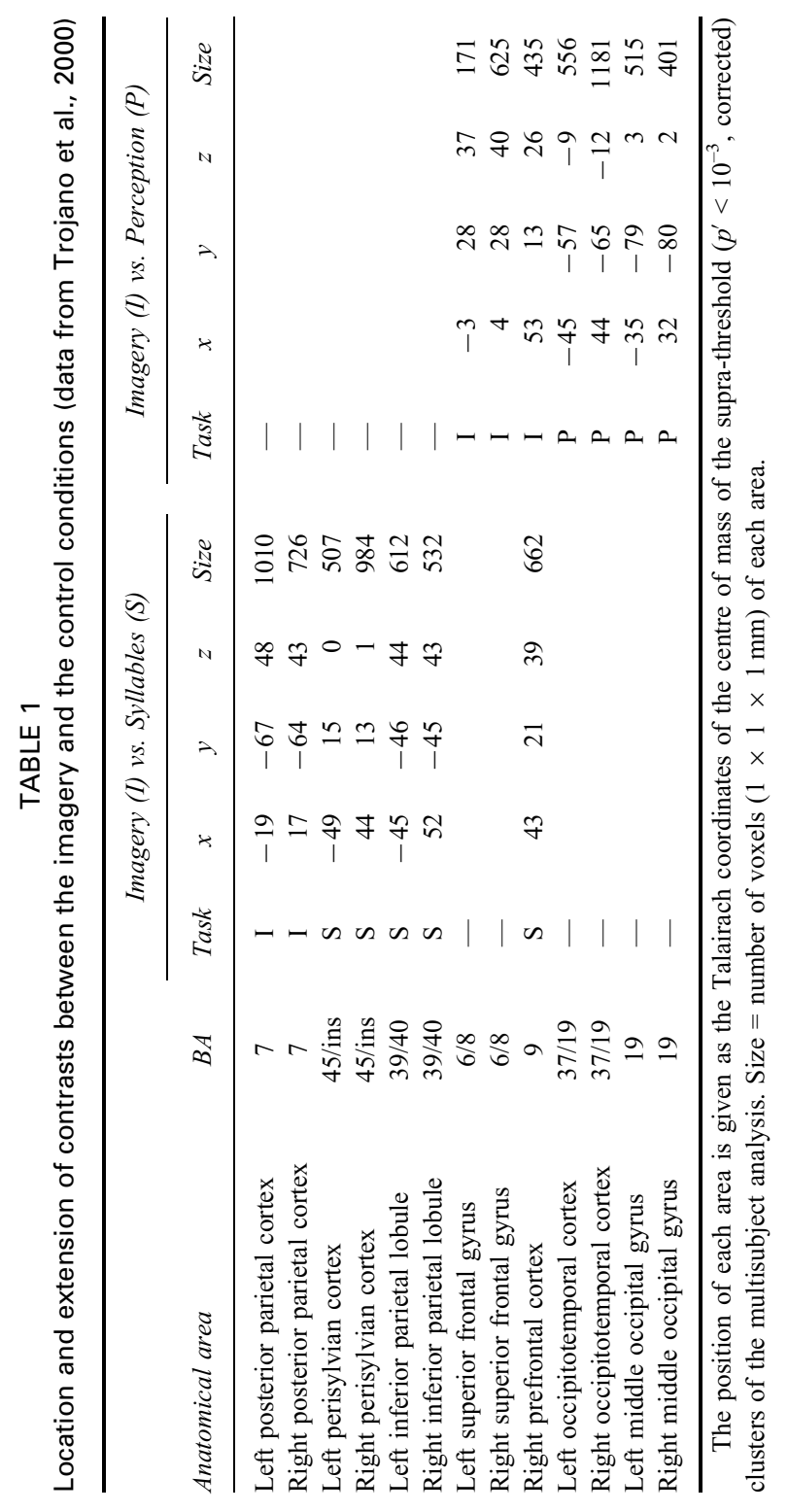


of the dorsal pathway. This dissociation lends further support to the view that the degree of recruitment of the cortical visual subsystems for mental imagery very much depends on the characteristics of the task, particularly the requirements on detailed image inspection, spatial transformation, and encoding of object properties (Thompson \& Kosslyn, 2000).

The results of our study of the mental clock task indicate that while there is convergence of the cortical processing streams for visuospatial processing in perception and imagery at the level of the dorsal pathway, the ventral pathway is recruited exclusively during the perceptual condition. The area in the superior PPC is active when the spatial task is performed on mental images, even in the absence of any visual stimulation. This similarity of activation patterns can be explained in two ways. First, the superior IPS might be instrumental in the computation of spatial transformations, regardless of whether the material is present in the visual field or merely as a mental image. Alternatively, any spatial transformation task, whether it involves visually perceived or imagined material, or indeed tactile stimulation (Sathian, Zangaladze, Hoffmann, \& Grafton, 1997) might require the implicit generation of mental visual representations (Kosslyn \& Sussmann, 1995).

\section{DO DIFFERENT KINDS OF SPATIAL IMAGERY TASKS RELY ON DIFFERENT CORTICAL NETWORKS?}

Our high-resolution fMRI findings demonstrated that posterior parietal cortex is strongly involved in the processing of spatially coded material in the imagery domain. The comparison of our results with those of recent studies of spatial transformations of visually presented material indicates that the analysis of visual space in perception and imagery has a common neural basis in the parietal lobes. It can be suggested that the neural networks involved in the processes of spatial processing are shared by several cognitive functions, including visuospatial imagery. In a subsequent study we aimed to clarify whether different parts of the PPC are involved in different kinds of spatial imagery tasks.

Current theoretical models predict that two different kinds of spatial encoding proceed in parallel. One process encodes discrete ("categorical") spatial relations, those easily described by verbal locatives like left/right or above/below, while the other encodes metric ("coordinate") spatial relations, representing precise, quantitative aspects of the spatial relationships (Kosslyn, 1994). Both cerebral hemispheres participate in spatial processing of visual input, but each seems to be specialised for a particular kind of spatial processing. The left hemisphere would be relatively faster than the right at encoding "categorical" spatial relations, while the right hemisphere would be superior at encoding metric ("coordinate") spatial relations (Brown \& Kosslyn, 1993; Kosslyn, 1987). It has been suggested that the categorical/coordinate dichotomy may 
apply also to the visual imagery domain (Kosslyn, Maljkovic, Hamilton, Horwitz, \& Thompson, 1995; Michimata, 1997).

The mental clock task as described above represents a paradigm for "coordinate" judgements in the imagery domain because it requires the generation of multipart mental images and a subsequent spatial metric comparison task (Michimata, 1997; Trojano \& Grossi, 1994). In a block-design experiment we contrasted the "coordinate" clock task with a "categorical" task applied to the same clock stimuli (Trojano et al., 2002). For the "categorical"' task we asked subjects to imagine analogue clock faces according to the procedure of the classical mental clock task. However, this time they had to judge whether both hands lay in a given half (upper, lower, right, or left) of the clock face. Both tasks share several cognitive processes: auditory processing of verbal instructions, image generation, image maintenance and scanning, and response selection procedures. The comparison between the two tasks, and that between each task and a third experimental condition employing the same auditorily presented verbal material and a comparable working memory load, should reveal whether the posterior parietal cortex is involved in both kinds of spatial processing and, moreover, whether any lateralisation of cortical activation corresponds to the categorical/coordinate dichotomy in the imagery domain (Michimata, 1997).

Seven healthy right-handed postgraduate students participated in the study. The experimental paradigm included the clock task given as in the previous study (coordinate condition). In the categorical condition, subjects were asked to imagine an analogue clock face showing the time verbally presented by the examiner. After each verbal presentation of a time, subjects heard a cue indicating one half of the clock face (left, right, upper, or lower; ISI = $1 \mathrm{~s}$ ) and they had to judge whether both hands lay in the cued half of the clock face (congruent trials) or not (noncongruent trials). For noncongruent trials, times were chosen that would correspond to a different cue. This choice was made to discourage subjects from resorting to verbal labels, and compel them to form mental images in response to all clock stimuli. We also included a nonspatial control condition, in which we asked subjects to count the syllables of each of the auditorily presented pairs of times and to report whether the total syllable number was odd or even. The three conditions were alternated in blocks of eight trials, and the categorical and the coordinate conditions were alternated in the sequence of stimulation conditions, with the control task always following the two imagery tasks. Subjects' responses were registered by an optic-fibre answer box and analysed for accuracy and response times. Subjects were asked to keep their eyes open during the scanning session and foveate a fixation cross in order to avoid eye movements.

MR hardware and sequences were the same as in the previous study. Data analysis, registration, and surface-based visualisation were performed with the fMRI software package BrainVoyager (Goebel \& Singer, 1999). The statistical analysis of the BOLD time courses, limited to cortical voxels, was based on the 
general linear model of the experiment with the categorical and the coordinate imagery tasks, and the nonspatial control condition (syllable counting) as the effects of interest. The global level of the signal time courses in each session was considered to be a confounding effect. Statistical results $\left(p^{\prime}<10^{-3}\right.$ corrected) were then visualised through projecting 3 -D statistical maps on surface reconstruction of the cortical sheet.

Analysis of behavioural data showed that the syllable counting task proved to be the most difficult task (mean correct reaction time: $2788 \pm 635 \mathrm{~ms} ; 76.2 \pm$ $12.7 \%$ of correct responses), while the categorical (mean correct reaction time: $2450 \pm 396 \mathrm{~ms} ; 84.8 \pm 3.9 \%$ of correct responses) and the coordinate (mean correct reaction time: $2413 \pm 416 \mathrm{~ms} ; 83.2 \pm 6.7 \%$ of correct responses) spatial judgement tasks yielded similar results. The three experimental conditions did not differ significantly in their behavioural measures (Kruskal-Wallis test). As for fMRI data, the contrasts between the categorical and the coordinate conditions and the control task yielded similar, but not overlapping, cortical activation patterns (Table 2).

Both spatial imagery tasks activated the superior parietal lobule bilaterally. Moreover, activated cortical areas were also seen along the anterior part of the intraparietal sulcus, extending into the inferior parietal lobule bilaterally in the coordinate task, and only on the left side in the categorical task. The relative contribution maps of the spatial imagery tasks (Figure 2) suggested that the categorical and the coordinate processing of spatial mental images shared a common region of activation in the superior parietal lobule, but with some differences between each other. The areas in the superior parietal lobule showed a relatively lateralised pattern of activation, with a higher (but not exclusive) contribution of the right side during the coordinate task, and a higher contribution of the left during the categorical task. Both tasks activated the angular gyrus bilaterally, in the depth of the anterior part of intraparietal sulcus; the

TABLE 2

Location and extension of contrasts between the coordinate and the categorical spatial imagery task (data from Trojano et al., 2002)

\begin{tabular}{|c|c|c|c|c|c|c|c|c|c|c|}
\hline & \multirow[b]{2}{*}{$B A$} & \multirow[b]{2}{*}{ Side } & \multicolumn{4}{|c|}{ Coordinate judgement } & \multicolumn{4}{|c|}{ Categorical judgement } \\
\hline & & & $x$ & $y$ & $z$ & Size & $x$ & $y$ & $z$ & Size \\
\hline \multirow[t]{2}{*}{ Superior parietal lobule } & 7 & $\mathrm{~L}$ & -17 & -71 & 53 & 67 & -15 & -71 & 49 & 624 \\
\hline & & $\mathrm{R}$ & 21 & -67 & 48 & 782 & 15 & -71 & 53 & 58 \\
\hline \multirow[t]{2}{*}{ Angular gyrus } & 40 & $\mathrm{~L}$ & -41 & -38 & 39 & 63 & -49 & -48 & 45 & 104 \\
\hline & & $\mathrm{R}$ & 37 & -45 & 45 & 64 & 46 & -47 & 44 & 56 \\
\hline Inferior frontal sulcus & $9 / 44$ & $\mathrm{R}$ & 37 & 13 & 38 & 136 & & & & \\
\hline
\end{tabular}

The position of each area is given as the Talairach coordinates of the centre of mass of suprathreshold clusters $\left(p^{\prime}<10^{-3}\right.$, corrected) of the group analysis. Size indicates number of voxels. 


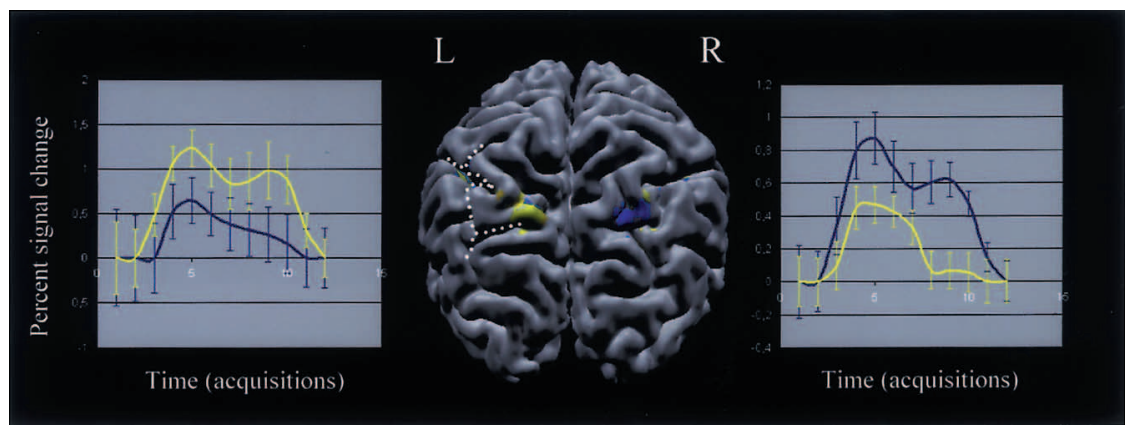

Figure 2. Multisubject GLM contrast maps $\left(p^{\prime}<10^{-3}\right.$, corrected) superimposed on a 3-D reconstruction of the cortical surface of an individual normalised 3-D anatomy from the second block-design study (Trojano et al., 2002). The figure (posterior view, left hemisphere on left side) shows the relative contribution (for an explanation see caption to Figure 1) map between the coordinate (in blue) and the categorical (in yellow) spatial tasks. The coordinate task produced a higher activation in the right PPC; the categorical task activated mainly the left PPC. Other foci of activation are present in the both angular gyrus bilaterally for both tasks and in the right prefrontal cortex for the coordinate task. As in the previous experiment (see Figure 1), no imagery-related activation was observed in occipitotemporal areas. The white dotted line corresponds to the intraparietal sulcus and its main branches.

The BOLD signal time courses from all the activated voxels in the PPC of each hemisphere, averaged across subjects and epochs, are shown on the respective side of the map (note that BOLD signal is plotted with different scales). On the left PPC the averaged signal time course reveals a higher percentage signal change in the categorical task (yellow line) than in the coordinate task (blue line); a reverse pattern is observed on the right side. Reprinted with permission of Elsevier Science (Trojano et al., 2002).

activation induced by the categorical task produced a larger cluster size on the left side. Moreover, the coordinate task specifically induced activation of the right prefrontal cortex encroaching upon the posterior end of the inferior frontal sulcus.

The analysis of single subjects' activations confirmed the robust finding that the superior parietal lobules were engaged in both spatial imagery tasks. Moreover, within the superior parietal lobules, four out of seven subjects showed larger left hemisphere activation in the categorical task than in the coordinate task, and five out of seven subjects showed larger right hemisphere activation in the coordinate task than in the categorical task. Considering the whole parietal lobes, the total number of activated voxels did not differ between the two hemispheres (left parietal lobe: $4786 \pm 1984$; right parietal lobe: $3852 \pm$ 1931; Wilcoxon $Z=1.16, p=.24$ ) or between the two tasks (coordinate task: $5268 \pm 2041$; categorical task: $3369 \pm 2262$; Wilcoxon $Z=1.54, p=.12$ ) across the seven subjects; the mean percentage number of activated voxels did not differ between the two tasks in the left parietal lobe (coordinate task: 46.3; categorical task: 53.7; Wilcoxon $Z=0.17, p=.87$ ), while it was significantly 
higher in the coordinate task (73.7) than in the categorical task (26.3) in the right parietal lobe (Wilcoxon $Z=2.03, p=.04$ ).

These findings confirmed that parietal lobes are strongly involved in the processing of spatially coded material in the imagery domain. Moreover, data pointed to a differential functional involvement of interconnected neural networks according to cognitive requirements of different spatial tasks. The coordinate judgement specifically relied on the activation of the right prefrontal cortex. In studies on visual and spatial mental imagery coactivation of frontal and parietal areas has often been reported and related to image generation or to image maintenance (Ishai, Ungerleider, \& Haxby, 2000; Mellet, Petit, Mazoyer, Denis, \& Tzourio, 1998; Thompson \& Kosslyn, 2000). Since behavioural results of our experiment demonstrate that the categorical and the coordinate imagery tasks had equivalent processing load, the differential activation of right prefrontal cortex could be explained by a higher processing load on spatial working memory during the coordinate task (Smith \& Jonides, 1999).

With respect to the issue of lateralisation of categorical and coordinate spatial judgements, Baciu, Koenig, Vernier, Bedoin, Rubin, and Segebarth (1999) showed a stronger activation of the left than of the right angular gyrus in a categorical task, and the reverse pattern of lateralisation in a coordinate task in the visual perception domain. Our findings are congruent with these data, but our whole-brain study allowed us to verify that some degree of relative lateralisation may also be found in regions other than the parietal cortex. In conclusion, the findings of this study support the idea that the superior parietal lobules are crucial for both categorical and coordinate spatial judgements. These regions, together with other parietal and prefrontal areas, showed a pattern of relative lateralisation, since the left hemisphere was more involved in the categorical task, while the coordinate task elicited activation of more extended regions of the right hemisphere and of right prefrontal regions thought to be involved in spatial working memory (Smith \& Jonides, 1999). The involvement of a frontoparietal network in the processing of mental images has been confirmed by recent fMRI studies on both spatial (Knauff, Kassubek, Mulack, \& Greenlee, 2000) and object (Ishai et al., 2000) imagery, but without any clear evidence of hemispheric asymmetry.

\section{IS IT POSSIBLE TO DIFFERENTIATE THE ROLE OF SINGLE NEURAL STRUCTURES IN DIFFERENT COGNITIVE COMPONENTS OF SPATIAL MENTAL IMAGERY?}

In functional neuroimaging like in psychometric studies (Michimata, 1997), it has to be considered that the observed differences in cortical activation might originate at different stages of the cognitive processes involved in the tasks. For example, it could be argued that the requirement of the coordinate judgement 
would elicit an image generation process more heavily dependent on the precise metric assembly of multi-part mental images, whereas the categorical judgement could induce a global, sketchier reconstruction of mental images. Alternatively, it could be hypothesised that generating a multipart mental image requires a metric spatially organised arrangement of its constituents, a cognitive step common to the two imagery tasks, and that the observed differences in cortical activation could arise during different spatial computations required by the categorical or the coordinate judgement. Novel experimental paradigms would be necessary to differentiate among the possible theoretical explanations of our findings. For instance, the coordinate/categorical distinction could be assessed in an experimental set including also two parallel visual perceptual conditions for the categorical and the coordinate judgement. However, this would imply several methodological drawbacks, since such perceptual tasks would be considerably easier than the imagery conditions, as has been stated in previous studies (French \& Painter, 1991). Other experimental approaches, for example by means of event-related fMRI paradigms, will be necessary to determine which cortical regions are involved in the generation of mental images, and which in subsequent spatial judgements.

Thompson and Kosslyn (2000) propose four types of imagery processing: image generation, image inspection, image maintenance, and image transformation. Most imagery tasks probably involve more than one if not all of these. Furthermore, some degree of sequential processing will also be required. In the case of the mental clock task, e.g., it can be argued that first an image of an analogue clock has to be generated on the basis of the information about the first time. This image has to be maintained while the second image is generated on the basis of the second time, then both images are inspected and compared, which might involve some spatial transformation of the images. Traditionally, the cortical representation of these components of the imagery system could only be probed in neuropsychological or functional neuroimaging studies by the comparison of different imagery tasks requiring a different contribution from these components. With the development of techniques for event-related fMRI, however, it has become possible to investigate the representation of component cognitive processes in imagery (and many other cognitive areas) within one task, and often at the level of the single trial.

We applied event-related fMRI to the same task that had been studied with the classical block design in the studies reported above (Formisano et al., 2000, 2002; Linden et al., 2000). In this series of event-related fMRI experiments, the mental clock task was administered to six subjects altogether. The MR hardware was the same as above. We used a BOLD sensitive single shot EPI sequence (echo time, $\mathrm{TE}=60 \mathrm{~ms}$; flip angle, $\mathrm{FA}=90^{\circ}$; matrix size $=64 \times 64$, voxel dimensions $=3 \mathrm{~mm} \times 3 \mathrm{~mm} \times 5 \mathrm{~mm}$ ) that permitted whole-brain imaging (16 slices) in $1.6 \mathrm{~s}$. Functional measurements were obtained every 2 s. Subjects again had to mentally construct analogue clocks from acoustically presented 
pairs of time and identify the greater angle while they kept the eyes open and directed on a fixation cross. Responses were indicated by button press with the right $(n=3)$ or left $(n=3)$ hand. Performance was again very good $(95 \%$ correct trials), and the reaction time was around $3 \mathrm{~s}$. Although the sampling rate was not much higher than the time required for the execution of the task ( $2 \mathrm{~s}$ vs. $3 \mathrm{~s})$, the fairly stable interval between acoustic stimulation and button press response permitted the analysis of the sequence activation of the different cortical areas recruited for the task.

The first area to be activated was the auditory cortex (Figure 3A), followed by the dorsolateral prefrontal cortex (DLPFC), the posterior parietal cortex, the supplementary motor area, and, finally, the motor cortex contralateral to the hand used for the button press. While the DLPFC activation was present during the entire interval between auditory and motor cortex activation and no hemispheric difference could be observed, the posterior parietal activity showed two peaks that were clearly separated in time, with a cluster that was activated early during the task and shows a bilateral distribution (but left predominance) and a late cluster that was confined to the right PPC. The duration of activation of the early cluster and the onset of the late right cluster correlated with reaction time.

It has been suggested above that the mental clock task requires the formation, maintenance, and spatial manipulation of images. We would expect areas involved in image generation to show a relatively early onset of BOLD activation, while areas involved in image manipulation and comparison would become active later because they presume the completion of a neuronal process in the image generation areas. Areas involved in the maintenance of the mental images should show a rise of activation at about the same time as the image generation areas but, unlike these, stay active during the entire trial. Of the three regions that were found to differ in their temporal characteristics during execution of the mental clock task each matched the criteria for one of the imagery subsystems. The early posterior parietal cluster with a bilateral, but predominantly left, distribution can be regarded as responsible for image generation. The DLPFC was active during the entire delay interval and thus matches the criteria for the image maintenance area, which is compatible with the well-known role of this area in the maintenance of visual information (Munk et al., 2002). The right posterior parietal cortex was activated last, which indicates its role in the spatial manipulation and analysis of the images. This result is in keeping with the large body of neuropsychological and neuroimaging literature that has indicated the particular importance of the right posterior parietal cortex for visuospatial processing, particularly when precise metric judgements are required or when analysis of local features of imagined visual objects is required (Ishai, Ungerleider, \& Haxby, 2002).

The present study, which was, to our knowledge, the first event-related functional neuroimaging study of mental imagery, could not address all aspects of the cerebral localisation of the subsystems of visuospatial imagery and their chronometry. In particular, the relationship of image inspection and spatial 


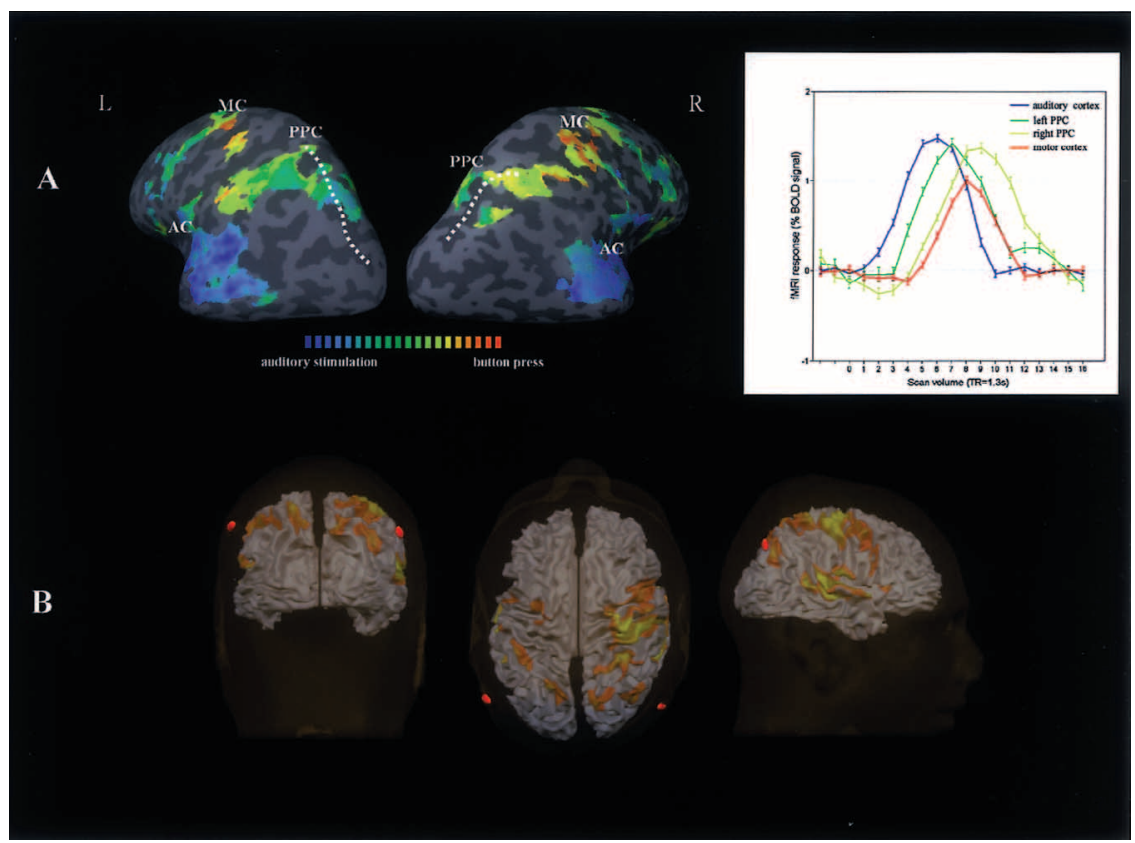

Figure 3. (A) Time-resolved multiple regression analysis of event-related fMRI time series. On the left, multisubject $(n=6)$ general linear model surface maps were superimposed on an inflated (lateral view) representation of the cortical sheet of a template brain normalised in Talairach space. The colour of significantly task-related voxels $\left(p^{\prime}<.001\right.$, corrected) encodes the latency of BOLD activation following the auditory presentation of the stimulus. Blue (red) colour indicates early (late) latencies of task-related activation corresponding to the auditory stimulation (motor response). Intermediate latencies of task-related activation are linearly represented according to the colour bar (Formisano et al., 2002). Dotted line indicates the intraparietal sulcus (posterior branch), PPC = posterior parietal cortex, $\mathrm{AC}=$ auditory cortex, $\mathrm{PFC}=$ prefrontal cortex, $\mathrm{MC}=$ motor cortex.

On the right, event-related BOLD responses of the auditory cortex, of the left and right posterior parietal cortex, and of the motor cortex during the execution of a single trial of the mental clock task. Reprinted with permission of Cell Press (Formisano et al., 2002).

(B) Head and cortex reconstruction from an anatomical MR data set of a single subject that shows the positions of the coil for rTMS (repetitive transcranial magnetic stimulation) stimulation in the left and right PPC. The rTMS coil was placed in correspondence of positions P3 and P4 of the international 10-20 EEG system. These positions were also marked using MR-visible vitamin E capsules (red spots on the skull) such that the relative displacement between fMRI activation in the PPC and sites of rTMS stimulation could be verified in individual subjects (see Sack et al., 2002).

analysis could not be clarified because, as in the previous block design studies, no significant activation of occipital and inferior temporal activation was observed during the mental clock task. The absence of imagery-related activation in early visual areas confirms the recent findings that certain imagery conditions, particularly those that rely on abstract patterns and schematic figures, produce activity in primary visual areas only to a small extent (Goebel et al., 1998a), or not at all (Mellet et al., 1996). 
For a better differentiation of the image inspection and spatial transformation mechanisms, the design of paradigms requiring the detailed analysis of a mental image and its subsequent spatial manipulation might be useful. For these and related questions the further development and refinement of the appropriate MR hardware, fast MR sequences, and analytical tools for event-related and singletrial fMRI techniques will be pivotal.

The functional relevance of PPC activity during the mental clock task was investigated by a further study (Sack et al., 2002) that combined functional neuroimaging with unilateral repetitive transcranial magnetic stimulation (rTMS). This study confirmed that visuospatial operations are associated with activation of the intraparietal sulcus bilaterally, but showed that only rTMS to the right parietal lobe induced an impairment of performance (Figure 3B). This functional parietal asymmetry in processing spatial mental images might indicate a capacity of the right parietal lobe to compensate for a lesion of the left. Such an explanation would be compatible with the current theories on spatial hemineglect that implicate an asymmetrical distribution of spatial attention (Mesulam, 1999). According to such models, the left hemisphere shifts attention in a contraversive direction while the right hemisphere directs attention in both directions (thus participating in a bilateral attentional network for the right hemispace); a lesion of the right hemispheric attention network would lead to hemineglect for the left hemispace, whereas a corresponding left hemispheric lesion could be compensated for by the preserved (right) hemisphere, which also covers the contralesional (right) hemispace.

\section{CONCLUSION}

The set of studies reviewed here converge to demonstrate that cortical areas in the PPC, along the intraparietal sulcus, are robustly involved in spatial mental imagery and in other tasks requiring spatial transformations. PPC is bilaterally involved in different kinds of spatial judgement. Yet the degree to which right and left PPC are activated in different tasks is a function of task requirements. From event-related AMRI data we obtained evidence that left and right PPC are activated asynchronously during the clock task and this could reflect their different functional role in subserving cognitive components of visuospatial imagery.

PrEview proof published online May 2004

\section{REFERENCES}

Alivisatos, B., \& Petrides, M. (1997). Functional activation of the human brain during mental rotation. Neuropsychologia, 35, 111-118.

Baciu, M., Koenig, O., Vernier, M. P., Bedoin, N., Rubin, C., \& Segebarth, C. (1999). Categorical and coordinate spatial relations: fMRI evidence for hemispheric specialization. Neuroreport, 10, $1373-1378$. 
Brown, H. D., \& Kosslyn, S. M. (1993). Cerebral lateralization. Current Opinion in Neurobiology, 3 , 183-186.

Cabeza, R., \& Nyberg, L. (2000). Imaging cognition II: An empirical review of 275 PET and fMRI studies. Journal of Cognitive Neuroscience, 12, 1-47.

Cohen, M. S., Kosslyn, S. M., Breiter, H. C., DiGirolamo, G. J., Thompson, W. L., Anderson, A. K., Brookheimer, S. Y., Rosen, B. R., \& Belliveau, J. W. (1996). Changes in cortical activity during mental rotation: A mapping study using functional MRI. Brain, 119, 89-100.

Di Salle, F., Formisano, E., Linden, D. E. J., Goebel, R., Bonavita, S., Pepino, A., Smaltino, F., \& Tedeschi, G. (1999). Exploring brain function with magnetic resonance imaging. European Journal of Radiology, 30, 84-94.

Farah, M. J., Hammond, K. M., Levine, D. N., \& Calvanio, R. (1988). Visual and spatial imagery: Dissociable systems of representation. Cognitive Psychology, 20, 439-462.

Formisano, E., Di Salle, F., Linden, D. E. J., Trojano, L., Grossi, D., Zanella, F. E., \& Goebel, R. (2000). Event-related fMRI during behaviorally controlled visuospatial imagery. NeuroImage, 11, S87.

Formisano, E., Linden, D. E. J., Di Salle, F., Trojano, L., Esposito, F., Sack, A. T., Grossi, D., Zanella, F. E., \& Goebel, R. (2002). Tracking the mind's image in the brain. I: Time-resolved fMRI during visuospatial mental imagery. Neuron, 35, 185-194.

French, C. C., \& Painter, J. (1991). Spatial processing of images and hemisphere function. Cortex, 27, 511-520.

Ghaem, O., Mellet, E., Crivello, F., Tzourio, N., Mazoyer, B., Berthoz, A., \& Denis, M. (1997). Mental navigation along memorized routes activates the hippocampus, precuneus, and insula. Neuroreport, 8, 739-744.

Goebel, R., Khorram-Sefat, D., Muckli, L., Hacker, H., \& Singer, W. (1998a). The constructive nature of vision: Direct evidence from functional magnetic resonance imaging studies of apparent motion and motion imagery. European Journal of Neuroscience, 10, 1563-1573.

Goebel, R., Linden, D. E. J., Lanfermann, H., Zanella, F. E., \& Singer, W. (1998b). Functional imaging of mirror and inverse reading reveals separate coactivated networks for oculomotion and spatial transformations. Neuroreport, 9, 713-719.

Goebel, R., \& Singer, W. (1999). Cortical surface-based statistical analysis of functional magnetic resonance imaging data. Neurolmage, 9, S64.

Grossi, D., Angelini, R., Pecchinenda, A., \& Pizzamiglio, L. (1993). Left imaginal neglect in heminattention: Experimental study with the o'clock test. Behavioral Neurology, 6, 155-158.

Grossi, D., Modafferi, A., Pelosi, L., \& Trojano, L. (1989). On the different roles of the two cerebral hemispheres in mental imagery: The "O'Clock test"' in two clinical cases. Brain and Cognition, $10,18-27$.

Ishai, A., Ungerleider, L. G., \& Haxby, J. V. (2000). Distributed neural systems for the generation of visual images. Neuron, 28, 979-990.

Ishai, A., Ungerleider, L. G., \& Haxby, J. V. (2002). Visual imagery of famous faces: Effects of memory and attention revealed by fMRI. NeuroImage, 16, S595.

Knauff, M., Kassubek, J., Mulack, T., \& Greenlee, M. W. (2000). Cortical activation evoked by visual mental imagery as measured by fMRI. Neuroreport, 11, 3957-3962.

Kosslyn, S. M. (1987). Seeing and imaging in the cerebral hemispheres: A computational approach. Psychological Review, 94, 148-175.

Kosslyn, S. M. (1994). Image and brain. Cambridge, MA: MIT Press.

Kosslyn, S. M., DiGirolamo, G. J., Thompson, W. L., \& Alpert, N. M. (1998). Mental rotation of objects versus hands: Neural mechanisms revealed by positron emission tomography. Psychophysiology, 3, 151-161.

Kosslyn, S. M., Maljkovic, V., Hamilton, S. E., Horwitz, G., \& Thompson, W. L. (1995). Two types of image generation: Evidence for left and right hemisphere processes. Neuropsychologia, 33, 1485-1510. 
Kosslyn, S. M., \& Sussmann, A. L. (1995). Roles of imagery in perception: Or, there is no such thing as immaculate perception. In M. Gazzaniga (Ed.), The cognitive neurosciences (pp. 1035-1042). Cambridge, MA: MIT Press.

Levine, D. N., Warach, J., \& Farah, M. (1985). Two visual systems in mental imagery: Dissociation of "what" and "where" in imagery disorders due to bilateral posterior cerebral lesions. Neurology, 35, 1010-1018.

Linden, D. E. J. (2002). Five hundred years of brain images. Archives of Neurology, 59, 308-313.

Linden, D. E. J., Formisano, E., Di Salle, F., Trojano, L., Zanella, F. E., Steinmetz, H., \& Goebel, R. (2000). Tracking the mind's image in the brain: Single trial fMRI during behaviourally controlled visuospatial imagery. European Journal of Neuroscience, 12(Suppl. 11), 86c.

Linden, D. E. J., Prvulovic, D., Formisano, E., Völlinger, M., Zanella, F. E., Goebel, R., \& Dierks, T. (1999). The functional neuroanatomy of target detection: An fMRI study of visual and auditory oddball tasks. Cerebral Cortex, 9, 815-823.

Luzzatti, C., Vecchi, T., Agazzi, D., Cesa-Bianchi, M., \& Vergani, C. (1998). A neurological dissociation between preserved visual and impaired spatial processing in mental imagery. Cortex, 34, 461-469.

Malach, R., Reppas, J. B., Benson, R. R., Kwong, K. K., Jiang, H., Kennedy, W. A., Ledden, P. J., Brady, T. J., Rosen, B. R., \& Tootell, R. B. (1995). Object-related activity revealed by functional magnetic resonance imaging in human occipital cortex. Proceedings of the National Academy of Sciences, USA, 92, 8135-8139.

Mellet, E., Petit, L., Mazoyer, B., Denis, M., \& Tzourio, N. (1998). Reopening the mental imagery debate: Lessons from functional anatomy. Neuroimage, 8, 129-139.

Mellet, E., Tzourio, N., Crivello, F., Joliot, M., Denis, M., \& Mazoyer, B. (1996). Functional anatomy of spatial mental imagery generated from verbal instructions. Journal of Neuroscience, $16,6504-6512$.

Mellet, E., Tzourio, N., Denis, M., \& Mazoyer, B. (1995). A positron emission tomography of visual and mental spatial exploration. Journal of Cognitive Neuroscience, 7, 433-445.

Mesulam, M. M. (1999). Spatial attention and neglect: Parietal, frontal and cingulate contributions to the mental representation and attentional targeting of salient extrapersonal events. Philosophical Transactions of the Royal Society of London: Biological Sciences, 354, 1325-1346.

Michimata, C. (1997). Hemispheric processing of categorical and coordinate spatial relations in vision and visual imagery. Brain and Cognition, 33, 370-387.

Milner, A. D., \& Goodale, M. A. (1995). The visual brain in action. Oxford, UK: Oxford University Press.

Munk, M. H. J., Linden, D. E. J., Muckli, L., Lanfermann, H., Zanella, F. E., Singer, W., \& Goebel R. (2002). Distributed cortical systems in visual short-term memory revealed by event-related functional magnetic resonance imaging. Cerebral Cortex, 12, 866-876.

O'Craven, K. M., \& Kanwisher, N. (2000). Mental imagery of faces and places activates corresponding stimulus-specific brain regions. Journal of Cognitive Neuroscience, 12, 1013-1023.

Paivio, A. (1978). Comparisons of mental clocks. Journal of Experimental Psychology: Human Perception, 4, 61-71.

Sack, A. T., Sperling, J. M., Prvulovic, D., Formisano, E., Goebel, R., Di Salle, F., Dierks, T., \& Linden, D. E. J. (2002). Tracking the mind's image in the brain. II: Transcranial magnetic stimulation reveals parietal asymmetry in visuospatial imagery. Neuron, 35, 195-204.

Sathian, K., Zangaladze, A., Hoffmann, J. M., \& Grafton, S. T. (1997). Feeling with the mind's eye. Neuroreport, 8, 3877-3881.

Smith, E. E., \& Jonides, J. (1999). Storage and executive processes in the frontal lobes. Science, 283, $1657-1661$.

Thompson, W. L., \& Kosslyn, S. M. (2000). Neural systems activated during visual mental imagery - a review and meta-analysis. In A. W. Toga \& J. C. Mazziotta (Eds.), Brain mapping: The systems (pp. 535-560). San Diego: Academic Press. 


\section{TROJANO ET AL.}

Trojano, L., \& Grossi, D. (1994). A critical review of mental imagery defects. Brain and Cognition, 24, 213-243.

Trojano, L., Grossi, D., Linden, D. E. J., Formisano, E., Goebel, R., Cirillo, S., Elefante, R., \& Di Salle, F. (2002). Coordinate and categorical judgements in spatial imagery: An fMRI study. Neuropsychologia, 40, 1666-1674.

Trojano, L., Grossi, D., Linden, D. E. J., Formisano, E., Hacker, H., Zanella, F. E., Goebel, R., \& Di Salle, F. (2000). Matching two imagined clocks: The functional anatomy of spatial analysis in the absence of visual stimulation. Cerebral Cortex, 10, 473-481.

Ungerleider, L. G., \& Haxby, J. V. (1994). "What" and "where"' in the human brain. Current Opinions in Neurobiology, 4, 157-165.

Ungerleider, L. G., \& Mishkin, M. (1982). Two cortical visual systems. In D. J. Ingle, M. A. Goodale, \& R. J. W. Mansfield (Eds.), Analysis of visual behavior (pp. 549-586). Cambridge, MA: MIT Press. 